\title{
Fatty liver- a silent disease
}

\section{Opinion}

Fatty liver or non alcoholic fatty liver disease is a term used to describe the accumulation of fat in the liver of people, who drink little or no alcohol.

Although the existence of some fat in liver can be normal, the accumulation can be a hidden threat to overall health. Non alcoholic fatty liver disease can take several forms - from harmless to lifethreatening.

These forms include in an increased severity condition:

a) Nonalcoholic fatty liver: A normal condition, not necessarily dangerous, with no significant complications. This condition is thought to be very common.

b) Nonalcoholic Steatohepatitis: In a small number of people with fatty liver, the fat will cause inflammation in the liver and as a consequence impaired liver function, leading to scarring of the liver- cirrhosis.

c) Nonalcoholic fatty liver disease-associated cirrhosis: When liver inflammation continuous, it can lead to scarring of the liver tissue. If scarring becomes severe, the liver could no longer function adequately, leading in liver failure.

Nowadays, fatty liver is a condition in the general population that is in increased at an alarming rate. The disease, at the last stage can be fatal, and paradoxically there can be no alcohol consumption. Before $30 y e a r s$, fat in liver was a rare diagnosis, but today can be characterize as a growing epidemic in adults. Unfortunately many people may do not even know that they suffer from it, until it is too late!

The main reason for this situation is the typical western diet, a diet rich in simple carbohydrates, white sugar and saturated fat. As an example, the French are famous for making the best foe grass worldwide through feeding by force their geese or ducks with sugar and starch. A very sad practice yet, with the terrific result of creating a fatty liver.

In the human body, sugar consumption triggers a "factory" which stores fat in the liver, a process known as adipogenesis, which is a normal response of the body to the sugar consumption. Moreover fructose sharply increases the answer to adipogenesis. This is a matter of fact, as today, corn syrup, a clean source of fructose, can be found in almost all the processed foods and agave syrup consist mainly of fructose and is considered to be a healthy substitute of sugar!

The overweight or obese people are the ones most at risk. The extra calories of nutrition are stored as fat in the liver, and when this procedure continues it can cause inflammation and scarring of the liver tissue, as it described above. This results in decreased organ operation in addition to increased risk of collapse. It is considered that the disease will be running out of control, as obesity rates continuously raise.

Although the individuals with increased body weight are the first "candidates" for the fatty liver, the disease can be observed even in people with normal weight. The individuals in high risk of
Volume 4 Issue 6 - 2016

\author{
Apergi Kiriaki \\ Clinical Dietician Nutritionist, Greece \\ Correspondence: Apergi Kiriaki, Clinical Dietician \\ Nutritionist, Greece, Tel 6987/21394, \\ Email ki.apergi@icloud.com \\ Received: August 10,2016 | Published: August 26, 2016
}

development of fatty liver are considered to be the ones with fat distribution around the waist, people with high cholesterol, high blood pressure, diabetes or pre-diabetes, even if their body weight is normal. Of course an increase in body fat is an important risk factor, as for every 22 extra pounds, the risk of fatty liver is increased by $10 \%$.

While a healthy liver is small and brown organ, when fat stores increase in volume, it becomes paler. If the liver continues to store up fat, the situation turns into "liver cirrhosis", even if the patient has never drink!

This disease is called as the "silent killer", because most people are asymptomatic. Symptoms may not be present or may not lead to the disease suspection, as they are not special for the disease. Possible symptoms include: fatigue, weight loss and poor appetite, nausea, abdominal pain in the right upper quadrant.

The good news is that this disease is able to manage, even be reversed, by the right nutrition. With the proper diet, fat accumulation in liver can remain stable and prevent from being develop into inflammation and cirrhosis. While liver cirrhosis is an irreversible condition, the previous situations can be reversed.

The condition can be suspected, during physical examination by a doctor, who may detect a liver enlargement in the upper right quadrant of the abdomen or accidentally through a simple blood test, by elevated liver enzymes. If the doctor suspects a liver disease, he should order further examination, usually by ultrasound or an MRI of the abdomen to examine the liver in detail.

Most patients with liver disease at an early stage are placed in a conventional therapeutic nutritional treatment, low in simple carbohydrates with a sufficient amount of protein and "good" anti inflammatory fatty acids, along with daily physical exercise and the prospect to lose weight, if needed.

After an about three- month follow up examination, if fat accumulation in liver shows signs of recession, the patient continues with the conservative therapy plan. If there are signs of aggravation or even stagnation, the doctor may order a liver biopsy to confirm the diagnosis and the presence of inflammation or scarring. 
For the prevention, as well as for the treatment of the disease, it is recommended an increased intake of food rich in vitamin $\mathrm{E}$ and anti inflammatory components, such as avocados, nuts, olive oil and green leafy vegetables. Alongside with the right diet, supplementation of $400 \mathrm{mg} /$ day of vitamin E may be needed, in addition to herbs like Milk Thistle, or components, such as lipoic acid, a potent antioxidant, and/ or N-acetyl-L-cystine, which help the liver compose an antioxidant enzyme called glutathione. Also the complex B vitamins and magnesium, will promote further "repair" and healing of the liver. ${ }^{1-7}$

\section{Acknowledgements}

None.

\section{Conflict of interest}

Author declares that there is no conflict of interest.

\section{References}

1. Non-alcoholic fatty liver disease (NAFLD). American College of Gastroenterology. 2013.
2. NAFLD. Non-alcoholic fatty liver disease. American Liver Foundation. 2013.

3. Feldman M. Sleisenger \& Fordtran's Gastrointestinal and Liver Disease: Pathophysiology, Diagnosis, Management. 9th ed. Philadelphia: Saunders Elsevier; 2013

4. Chalasani N, Younossi Z, Lavine JE, et al. The diagnosis and management of non-alcoholic fatty liver disease: Practice guideline by the American Gastroenterological Association, American Association for the Study of Liver Diseases and American College of Gastroenterology. Gastroenterology. 2012;142(7):1592-1609.

5. Golden AK. Decision Support System. Mayo Clinic. Rochester, USA; 2013.

6. Ludwig J, Viggiano TR, McGill DB, et al. Nonalcoholic steatohepatitis: Mayo Clinic experiences with a hitherto unnamed disease. Mayo Clin Proc. 1980;55(7):434-438.

7. Venkatesh SK, Yin M, Ehman RL. Magnetic resonance elastography of liver: Technique, analysis and clinical applications. J Magn Reson Imaging. 2013;37(3):544-555. 